\title{
Intra-Articular Therapies in the Treatment of Hand Osteoarthritis: A Systematic Literature Review
}

\author{
Féline P. B. Kroon ${ }^{1} \cdot$ Roxana Rubio $^{1} \cdot$ Jan W. Schoones $^{2} \cdot$ Margreet Kloppenburg $^{1,3}$
}

Published online: 9 December 2015

(c) The Author(s) 2015. This article is published with open access at Springerlink.com

\begin{abstract}
Background Local treatments to alleviate symptoms in hand osteoarthritis (OA) are preferred, especially in elderly patients with comorbidities. Therefore, we have summarized the benefits and harms of intra-articular (IA) therapies.

Methods We conducted a systematic literature review until May 2015, including all controlled trials investigating efficacy or safety of any IA therapy in carpometacarpal (CMC) and interphalangeal (IP) OA compared with placebo or other treatments. Two authors independently selected trials and assessed risk of bias using the Cochrane tool. The main efficacy outcome was pain. We performed meta-analysis where appropriate.

Results A total of 13 trials (864 patients) studying CMC $(n=11)$ and IP OA $(n=2)$ were included, comparing corticosteroids or hyaluronic acid (HA) versus placebo ( $n=4$ and $n=3$ ), and corticosteroids versus HA $(n=6)$. Single studies investigated infliximab, dextrose, and different HAs. The overall risk of bias was unclear or high in most trials. Meta-analysis of two trials comparing
\end{abstract}

Electronic supplementary material The online version of this article (doi:10.1007/s40266-015-0330-5) contains supplementary material, which is available to authorized users.

Féline P. B. Kroon

f.kroon.reum@lumc.nl

Margreet Kloppenburg

g.kloppenburg@lumc.nl

1 Department of Rheumatology, Leiden University Medical Center, PO Box 9600, 2300 RC Leiden, The Netherlands

2 Walaeus Library, Leiden University Medical Center, Leiden, The Netherlands

3 Department of Clinical Epidemiology, Leiden University Medical Center, Leiden, The Netherlands corticosteroids with placebo in CMC OA showed no improvement in pain [mean difference $-3.56,95 \%$ confidence interval (CI) -13.87 to 6.75 , scale $0-100$ ). HA also appeared not efficacious compared with placebo in CMC OA. One trial comparing corticosteroids with placebo in IP OA demonstrated significantly improved pain during movement. No convincing evidence for efficacy of corticosteroids or HA over the other or alternative therapies was found. Only local adverse events were reported. No specific IA therapy appeared more harmful than another.

Conclusion Despite a beneficial short-term safety profile, IA corticosteroids or HA do not appear more effective than placebo in CMC OA. The suggestion that IA corticosteroids might be efficacious in IP OA requires confirmation.

\section{Key Points}

Despite a beneficial short-term safety profile, intraarticular corticosteroids or hyaluronic acid do not appear more effective than placebo in thumb-base osteoarthritis.

Intra-articular corticosteroids might be efficacious in interphalangeal osteoarthritis, although this finding requires confirmation.

\section{Introduction}

Hand osteoarthritis (OA) is a prevalent joint disorder with a high burden of disease, leading to pain, loss of function and a decreased quality of life [1-3]. The prevalence of 
symptomatic hand $\mathrm{OA}$ among men and women aged $>70$ years was estimated to be 13 and $26 \%$, respectively, in a large population-based cohort study [3], and the prevalence of radiographic hand $\mathrm{OA}$ is even higher, affecting up to 55 and $67 \%$ of men and women over the age of 55 years [1]. No treatments are yet available to modify the disease course [4]. The main aim for therapies in hand $\mathrm{OA}$ is therefore to alleviate symptoms. For this purpose, non-pharmacological treatments (e.g. education, the use of assistive devices, and the application of splints for thumb base OA) as well as pharmacological treatment modalities [most importantly simple analgesics like paracetamol and non-steroidal anti-inflammatory drugs (NSAIDs)] are available. The European League Against Rheumatism (EULAR) recommends a combination of nonpharmacological and pharmacological treatment modalities for the optimal management of hand OA, with a preference for local treatments over systemic treatments [5]. Local treatment could be an attractive treatment modality, especially in elderly patients with more comorbidities.

A well-known, widely used form of local treatment is the injection of corticosteroids into the affected joint, particularly for patients with thumb-base OA [6]. The local antiinflammatory effects of corticosteroid injections are hypothesized to decrease inflammation and consequently relieve the symptoms of OA. However, important side effects like infectious arthritis and cartilage damage have been described, although these adverse events are infrequent [7]. The EULAR 2007 recommendations advocate the use of intra-articular injection of corticosteroids to alleviate painful flares of OA, especially in thumb-base OA, based on expert opinion [5]. However, this recommendation was not supported by the 2012 American College of Rheumatology (ACR) recommendations for the management of OA, which "conditionally recommends not using intra-articular corticosteroids" in thumb-base OA [8].

Another well-known intra-articular treatment is the injection of hyaluronic acid in the osteoarthritic joint (commonly called viscosupplementation). Viscosupplementation is based on the physiologic importance of hyaluronan in the synovial fluid, and it is hypothesized that this can restore the reduced viscoelasticity of the synovial fluid in osteoarthritic joints and thus decrease pain, improve mobility and restore the natural protective functions of hyaluronan in the joint [9, 10]. Reported side effects include local reactions, but also more serious side effects like pseudoseptic reactions and flare-ups of the disease $[11,12]$. Experts do not yet agree on the usefulness of this form of intra-articular therapy, since EULAR guidelines specify that intra-articular hyaluronan may be useful in treating thumb-base OA, whereas ACR guidelines conditionally recommend not using intra-articular hyaluronates $[5,8]$.
A recent systematic review assessing the efficacy of intra-articular injections of corticosteroids and hyaluronic acid in thumb-base OA concluded that intra-articular hyaluronic acid may be useful to increase functional capacity and that intra-articular corticosteroids can decrease pain in thumb OA after 24 weeks [13]. However, the authors found large heterogeneity in the results of their metaanalysis. Moreover, they did not include other subsets of OA, e.g. interphalangeal (IP) OA and erosive OA, and excluded trials that assessed other intra-articular therapies besides corticosteroids or hyaluronic acid.

The aim of the present study was to assess the benefits and harms of all forms of intra-articular therapies in the treatment of hand OA, including all its subsets.

\section{Methods}

We conducted a systematic literature review, including all published randomised controlled trials (RCTs) and quasiRCTs (i.e. where allocation was not truly random), without language restrictions. We included trials of adults with hand OA, regardless of its subset (including thumb-base OA, IP OA and erosive OA), as determined by clinical diagnosis or fulfilment of the ACR criteria for hand OA [14]. Studies including participants with other diseases were only eligible if the results from participants with hand OA were presented separately. We included studies that compared any form of intra-articular therapy in the hand joints (including, but not restricted to, corticosteroids and hyaluronic acid) versus placebo; another intra-articular therapy; another pharmacological therapy; a non-pharmacological treatment; or different doses, frequency or duration of the same therapy. Studies that did not assess any efficacy or safety outcomes were not eligible for inclusion.

\subsection{Search Strategy}

We searched the following databases up to May 2015: PubMed, Embase, the Cochrane Library, the Cochrane Central Register of Controlled Trials (CENTRAL), Web of Science, Cumulative Index to Nursing and Allied Health Literature (CINAHL), Academic Search Premier and ScienceDirect. The complete search strategy is provided in the Electronic Supplementary Material (ESM). Additionally, we searched three clinical trial registries to identify ongoing and recently finished studies [ClinicalTrials.gov, the World Health Organization (WHO) International Clinical Trials Registry Platform (ICTRP) search portal and the International Standard Randomised Controlled Trial Number (ISRCTN) registry]. We contacted authors of completed but still unpublished trials that were identified in clinical trial registries to request more information about 
their study. We also screened conference abstracts of the EULAR, ACR and OsteoArthritis Research Society International (OARSI) annual conferences from the last 5 years, as well as reference lists from included studies and other systematic reviews on the safety and efficacy of intra-articular therapies for hand OA. We contacted authors of eligible studies that were published only as a conference abstract for additional information, and excluded conference abstracts published more than 5 years ago.

\subsection{Study Selection and Data Collection}

Two review authors (FK, RR) independently screened the retrieved titles and abstracts, and full-text papers were retrieved and read if necessary to determine inclusion. Disagreements between the authors were discussed in a consensus meeting. In case of non-consensus, a third reviewer (MK) decided whether the study was eligible. One review author (FK) extracted data from each included study regarding study design, study duration, characteristics of the study population, interventions, outcome measures, timing of outcome assessment, co-interventions, results for outcomes of interest, losses to follow-up and funding. A second review author (RR) verified extracted data.

\subsection{Assessment of Risk of Bias}

Two review authors (FK, RR) independently assessed the risk of bias of each included study with regard to random sequence generation, allocation concealment, blinding (of participants, care provider and outcome assessor), incomplete outcome data, selective outcome reporting and other sources of bias according to the Cochrane risk of bias tool [15]. Each criterion was judged as 'low risk of bias', 'high risk of bias' or 'unclear' (either lack of information or uncertainty over the potential for bias). In addition, an 'overall assessment' was formed for each study, based on the individual judgements for each risk of bias item. Items assessing selection bias and blinding were considered 'key domains', i.e. the most important domains in the risk of bias of a study. Studies were judged as having an overall high risk of bias if one or more items from the 'key domains' were judged as high risk. In cases where both key domains were judged as unclear risk, that study was rated as having an overall high risk of bias when more than two of the three remaining items were both rated high or unclear, and it was rated as having an overall unclear risk of bias when two of the remaining items were rated as low and unclear risk or if at least two items were both rated as low risk. In cases where both key domains were judged as low risk, that study was rated as having an overall unclear risk of bias when more than two remaining items were both rated high or unclear, and it was rated as having an overall low risk of bias when two of the remaining items were rated as low and unclear risk or if at least two items were rated as low risk. Disagreements were discussed, and in case of non-consensus a third review author (MK) made the final decision.

\subsection{Outcome Measures}

All outcomes were assessed at baseline, week 4, week 12 and week 24. The main efficacy outcome was pain on a visual analogue scale (VAS) or a numerical rating scale (NRS). Other efficacy outcomes were self-reported physical function, patient global assessment, joint activity, health-related quality of life and hand strength, according to the core domains in clinical trials for hand OA proposed by Outcome Measures in Rheumatology (OMERACT) [16]. The safety outcomes that were assessed included the number of withdrawals due to adverse events, the number of (local or systemic) adverse events and the number of serious adverse events.

\subsection{Data Analysis}

Meta-analysis was only performed if the study data were clinically and statistically sufficiently homogeneous and/or the risk of bias was judged to be low enough to produce reliable results, using Review Manager 5.3 statistical software. For studies judged as clinically homogeneous with respect to intervention groups, control groups, timing of outcome assessment and outcome measures, the $I^{2}$ statistic was used to test for statistical heterogeneity. For continuous outcomes, mean differences (MDs) with corresponding $95 \%$ confidence intervals (CIs) were calculated, unless different scales were used to measure the same conceptual outcome (e.g. pain), in which case the standardised mean difference (SMD) was calculated with corresponding $95 \%$ CIs. SMDs are calculated by dividing the MD by the standard deviation (SD), resulting in a unitless measure of effect. For dichotomous outcomes, the results were expressed as risk ratios (RRs) with corresponding $95 \%$ CIs. A random-effects model was used as the default option to be conservative in all analyses, independent of the $I^{2}$. The sample mean and SD was estimated from studies reporting only the median and (interquartile) range, using the appropriate approximation method proposed by Wan et al. [17]. Studies that could not be included in the meta-analysis were described narratively. 


\section{Results}

\subsection{Description of Included Studies}

Database searching initially identified 2157 records, of which 24 qualified for full review, and 13 studies were finally included (see Fig. 1) [18-31]. We additionally identified 42 studies in clinical trial registries, of which two were eligible for inclusion. However, both trials were finally excluded as, upon request for more information from the authors, one trial appeared to be terminated prematurely due to adverse events in the intervention group, and the other trial was completed but results were not published because the pharmaceutical company did not pursue the indication. No additional records were found through other sources. All disagreements between the authors (FK, RR) on article selection $(n=6)$ were resolved in a consensus meeting.

A total of 13 studies involving 864 participants (range 20-200, median 60), published between 2004 and 2014 were included. The mean age of participants was 62.8 years (reported in 12 trials). Four studies only included women, and in the other nine studies $84.5 \%$ of the participants were women. Most studies $(n=11)$ only included participants with carpometacarpal (CMC) OA, and two trials only included participants with IP OA [20, 30], of which one study specifically included participants with erosive OA [20]. In many studies $(n=11)$, radiographic evidence of OA had to be present on top of clinical signs and symptoms.
Four trials compared corticosteroids with placebo; three trials compared hyaluronic acid with placebo; six studies compared corticosteroids with hyaluronic acid; and single studies compared infliximab with placebo, corticosteroids with dextrose, different frequencies of hyaluronic acid injections and hyaluronic acid with a low versus a high molecular weight (Table 1). A total of 280 participants were treated with intra-articular corticosteroid injections: three trials used triamcinolone acetonide (dose range $10-40 \mathrm{mg}$ in $0.5-1 \mathrm{ml}$, injected once in all studies) $[18,21$, 24], two studies used triamcinolone hexacetonide (dose range $4-6 \mathrm{mg}$ in $0.2-0.3 \mathrm{ml}$, injected once in both studies) $[26,30]$, two studies used betamethasone $(3 \mathrm{mg}$ in $0.5 \mathrm{ml}$ injected three times in one trial, and a single injection of an unknown dose in $1 \mathrm{ml}$ in the other) [22, 27], and two studies used methylprednisolone $(40 \mathrm{mg}$ in $0.5-1 \mathrm{ml}$ injected once in both studies) [23, 31]. Two trials added a small dose of lidocaine $2 \%$ to the injection fluid $[23,30]$. A total of 360 participants were treated with intra-articular hyaluronate injections: three studies used Hylan G-F 20 (8 $\mathrm{mg}$ in $1 \mathrm{ml}$, injected once or twice) [19, 22, 24], six studies used sodium hyaluronate (dose range $5-15 \mathrm{mg}$ in $0.5-1 \mathrm{ml}$, injected one to three times) $[18,21,25,27-29$, 31]. A total of 172 participants were treated with placebo, comprising a saline injection of $0.2-1 \mathrm{ml}$ in four studies $[19,20,22,26]$ or a local anaesthetic in two studies $(0.1 \mathrm{ml}$ lidocaine $2 \%$ or $0.5 \mathrm{ml}$ bupivacaine $0.5 \%$ ) [24, 30]. Finally, 30 participants in one trial were treated with intraarticular dextrose $20 \%$ injections $(0.5 \mathrm{ml}$, combined with
Fig. 1 Study selection process. ICTRP International Clinical Trials Registry Platform, ISRCTN International Standard Randomised Controlled Trial Number, $W H O$ world Health Organization

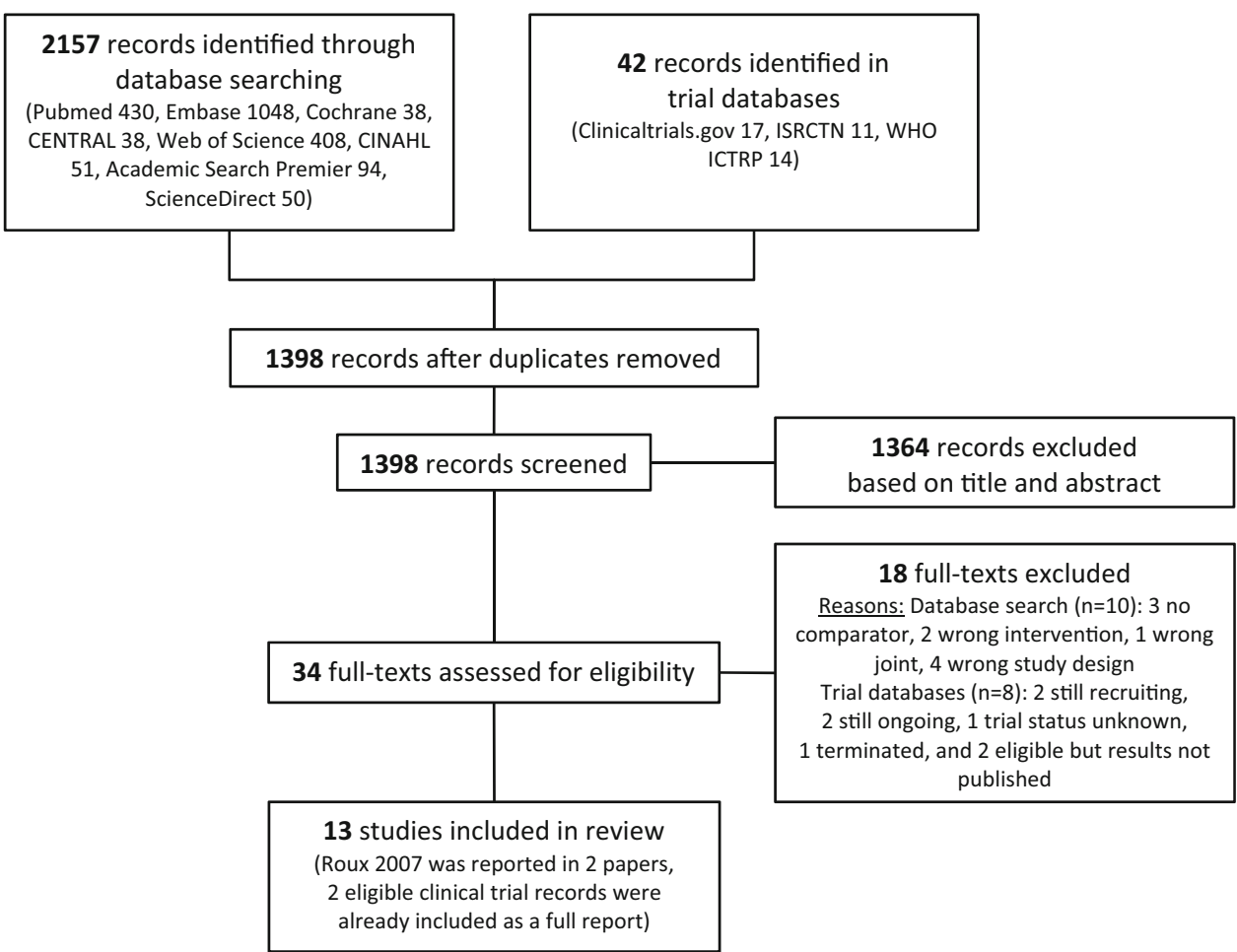




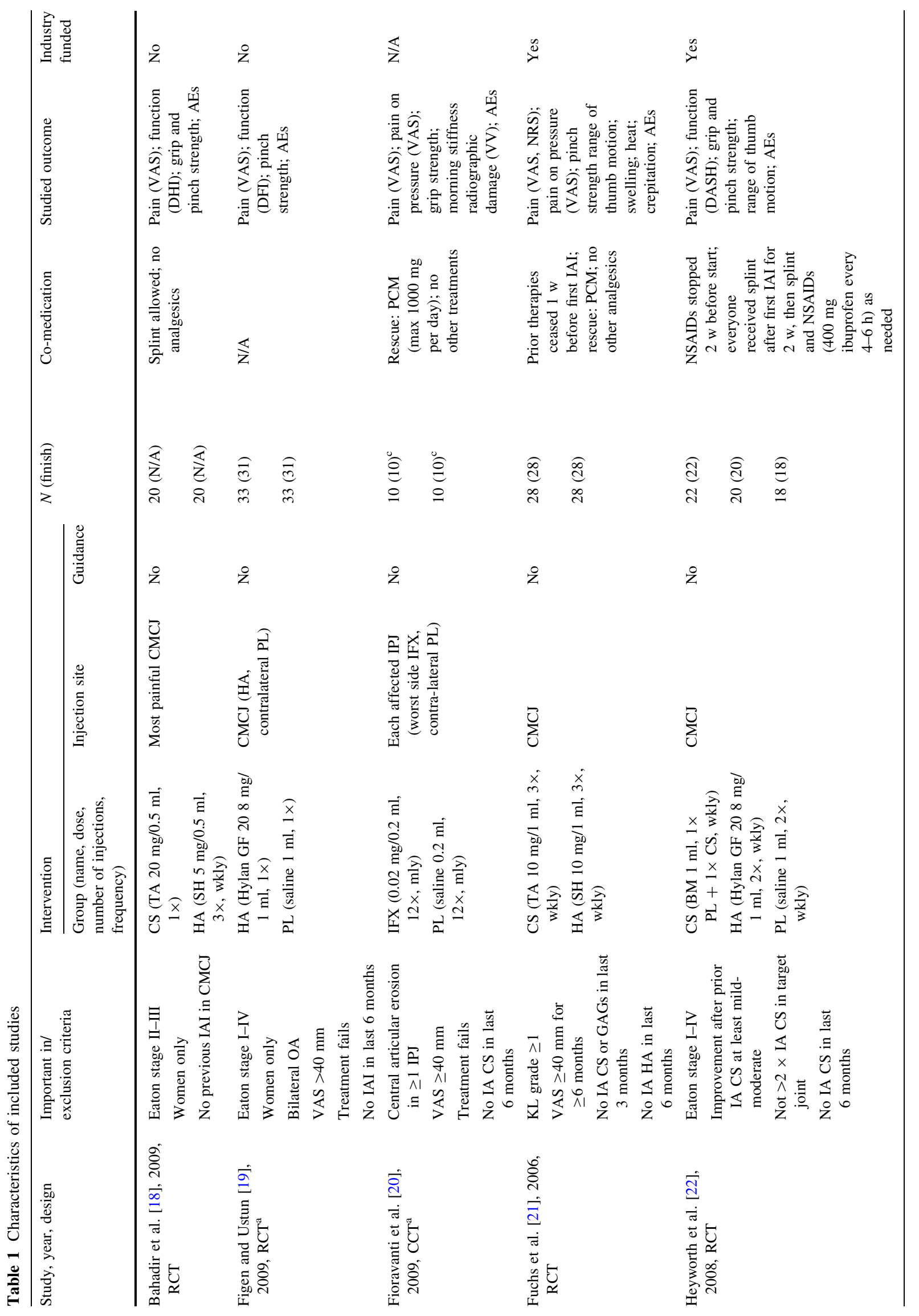




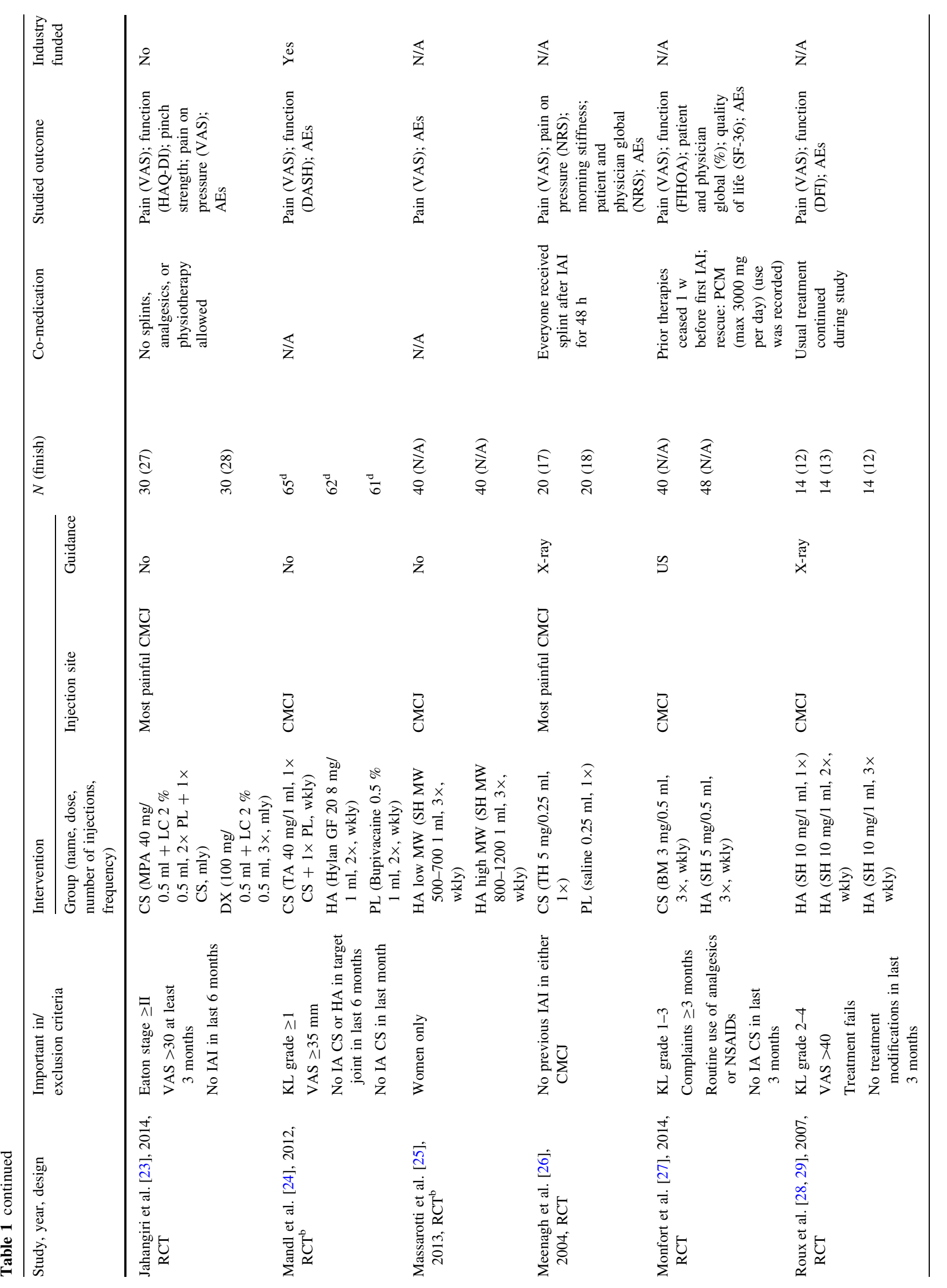




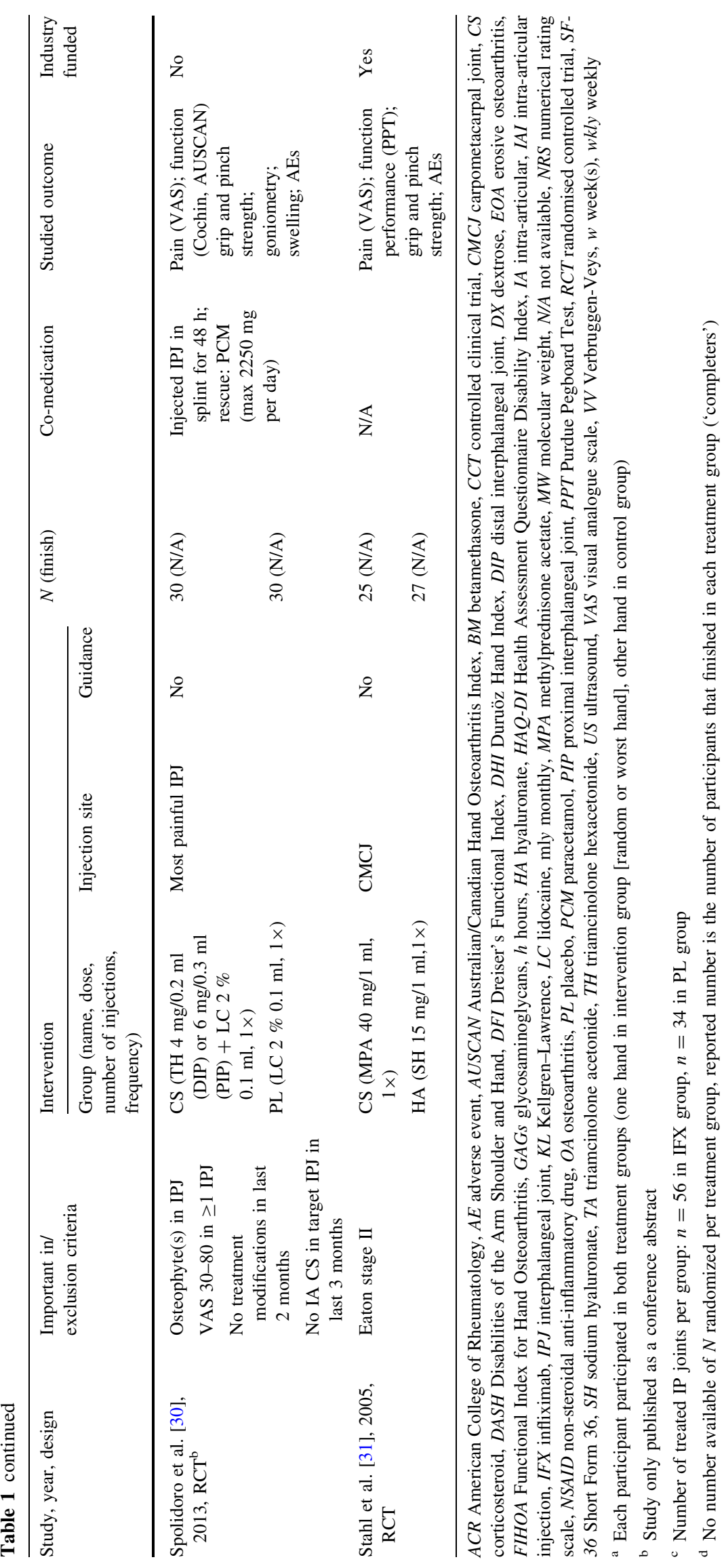




\begin{tabular}{|c|c|c|c|c|c|c|c|c|}
\hline & 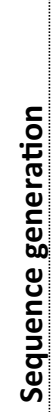 & 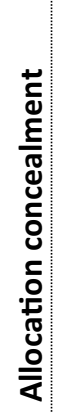 & $\begin{array}{c}\frac{0}{2} \\
\frac{0}{0} \\
\frac{0}{0} \\
\frac{0}{0} \\
\frac{0}{00} \\
\frac{0}{0} \\
\frac{.0}{0}\end{array}$ & 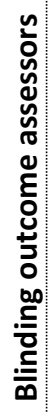 & 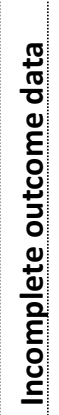 & 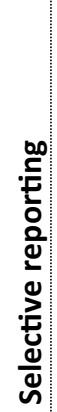 & $\begin{array}{l}\frac{y}{0} \\
\frac{0}{0} \\
\frac{1}{0}\end{array}$ & 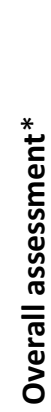 \\
\hline Bahadir[18] & $?$ & $?$ & $\boldsymbol{\theta}$ & + & $?$ & + & + & $\theta$ \\
\hline Figen[19] & $\theta$ & $?$ & ? & $?$ & $\theta$ & $\boldsymbol{\theta}$ & $\theta$ & $\theta$ \\
\hline Fioravanti[20] & $\boldsymbol{\theta}$ & $\boldsymbol{\theta}$ & + & + & + & + & $\theta$ & $\theta$ \\
\hline Fuchs[21] & $?$ & $?$ & $\theta$ & + & + & $\theta$ & $t$ & $\theta$ \\
\hline Heyworth[22] & + & + & + & + & $?$ & $\theta$ & $\boldsymbol{\theta}$ & $?$ \\
\hline Jahangiri[23] & + & + & $t$ & $t$ & $?$ & $\theta$ & $\theta$ & $?$ \\
\hline Mandl[24] & $?$ & $?$ & $?$ & $?$ & $?$ & + & + & $?$ \\
\hline Massarotti[25] & ? & ? & ? & ? & ? & + & + & $?$ \\
\hline Meenagh[26] & + & + & + & + & + & $?$ & $?$ & $t$ \\
\hline Monfort[27] & ? & ? & $\theta$ & + & $?$ & + & $\boldsymbol{\theta}$ & $\theta$ \\
\hline $\operatorname{Roux}[28,29]$ & $?$ & $?$ & $\theta$ & $?$ & + & + & + & $\theta$ \\
\hline Spolidoro[30] & + & $t$ & $t$ & + & + & $\boldsymbol{\theta}$ & $t$ & + \\
\hline Stahl[31] & $\theta$ & $?$ & $?$ & $?$ & $?$ & $?$ & ? & $\theta$ \\
\hline
\end{tabular}

Fig. 2 Risk of bias summary: review author's judgement about each risk of bias item for each included study ( + indicates low risk of bias, - indicates high risk of bias, ? indicates unclear risk of bias, * indicates overall assessment based on the individual judgements for each risk of bias item)

$0.5 \mathrm{ml}$ lidocaine $2 \%$, injected three times monthly) [23], and ten participants were treated with intra-articular infliximab injections $(0.2 \mathrm{ml}, 0.1 \mathrm{mg} / \mathrm{ml}$, injected 12 times monthly) [20].

\subsection{Risk of Bias}

Most trials were assessed as being at high or unclear risk of bias for multiple items (Fig. 2). In the overall assessment, only two studies were judged as having a low risk of bias $[26,30]$. Four trials were at low risk of selection bias, as they described adequate sequence generation and allocation concealment; the risk of selection bias in the other trials was high $(n=3)$ or unclear $(n=6)$. One additional trial was at high risk of selection bias even though sequence generation and allocation concealment were performed using methods associated with a low risk of bias, since this study selectively included participants based on the probability of a positive treatment effect (judged as high risk of bias in the item 'other bias') [22]. Participants were adequately blinded in five trials; however, in the remaining studies, participants were not blinded $(n=4)$ or it was unclear whether participants were blinded $(n=4)$, although most trials $(n=8)$ adequately blinded the outcome assessor(s). One trial had unexplained incomplete outcome data and was assessed as being at high risk of attrition bias. The risk of attrition bias remained unclear in seven trials, for example because the number of participants finishing the study was not described $(n=5)$. Many trials had a high risk of reporting bias $(n=5)$, e.g. as they did not report all assessed outcomes at all time points or only reported $p$ values. Other potential sources of bias were identified in five trials and included inappropriate statistical analyses for the selected study design $(n=2)$ $[19,20]$, exclusion of participants after inclusion [23] or after treatment allocation [27], and selectively including patients based on probability of a positive treatment effect $(n=2)[20,22]$.

\subsection{Effects of Interventions}

Most studies were clinically too heterogeneous to compare, did not provide data eligible for meta-analysis (e.g. data only presented in figures, or presentation of point estimates without a measure of variance), and/or the risk of bias was judged to be too high to produce reliable results. Only results of two studies, comparing corticosteroid injections versus placebo in participants with CMC OA, could be pooled $[24,26]$. The remaining studies are discussed narratively under each relevant comparison. A summary of the most important findings is presented in Table 2.

\subsubsection{Intra-Articular Corticosteroids Versus Placebo}

Three studies $(n=206)$ compared a corticosteroid injection with placebo in participants with CMC OA [22, 24, 26]. Pooled results from two studies $(n=166$, unclear and low risk of bias) for our primary outcome measure pain on VAS showed no difference up to 26 weeks between intraarticular corticosteroids and placebo [MD -3.56 (95\% CI -13.87 to 6.75$)$ on a $100-m m$ VAS; Fig. 3]. The third study ( $n=40$, unclear risk of bias) concluded that both groups showed a decrease in pain compared with baseline, although there were no significant between-group differences at any of the time points up to 26 weeks (data only presented graphically). Also, for the other reported efficacy outcomes [i.e. function (two trials), grip strength (one trial), pinch strength (one trial), pain on joint palpation (one trial), joint stiffness (one trial), patient global assessment (one trial), physician global assessment (one trial) and range of motion (one trial)], no between-group differences up to 26 weeks were found by any study in this comparison. No adverse events were observed in any of the treatment groups in two trials, although one trial [24] observed 


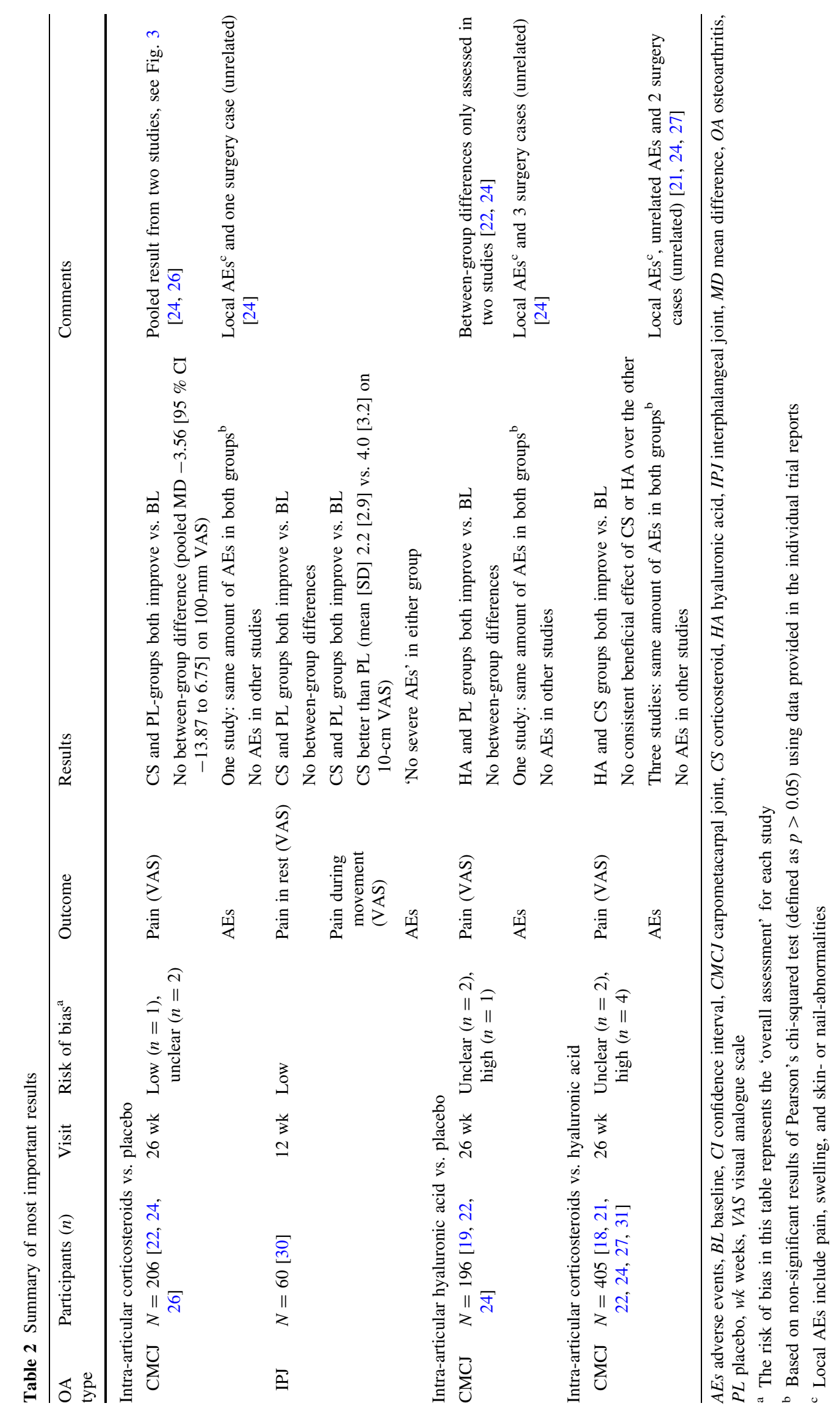




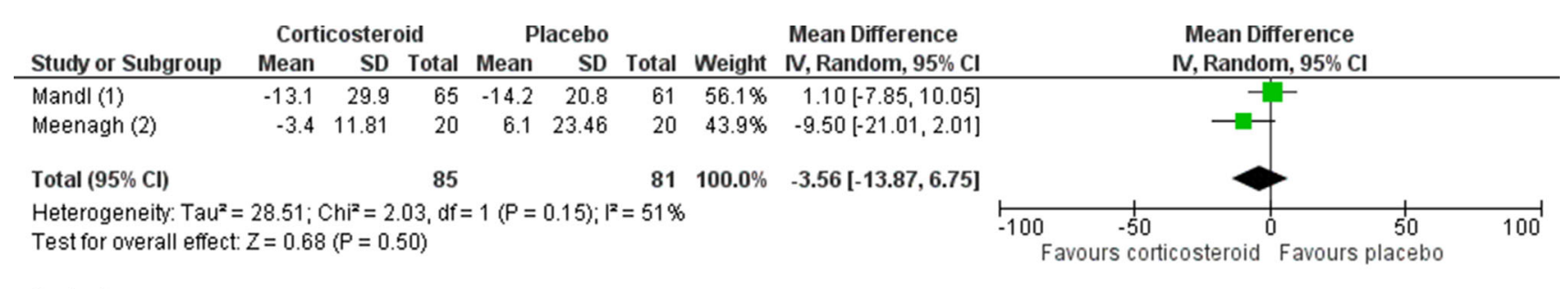

\section{Footnotes}

(1) 26 weeks, VAS $0-100 \mathrm{~mm}$, higher is worse

(2) 24 weeks, VAS $0-100 \mathrm{~mm}$, higher is worse

Fig. 3 Forest plot: comparison of intra-articular corticosteroids versus placebo in participants with thumb-base OA, outcome pain on VAS. The studies included in the forest plot were Mandl et al. [24]

adverse events in both treatment groups [9/65 in the corticosteroid group (all local adverse events such as pain, swelling and skin or nail abnormalities) and $3 / 61$ in the placebo group (of which two were local adverse events and one was a surgery unrelated to study medication)].

Spolidoro et al. [30] ( $n=60$, low risk of bias) studied participants with IP OA and found a decrease in pain compared with baseline for both groups, although there were no between-group differences for pain at rest at any of the time points up to 12 weeks. However, pain during joint movement improved more in the corticosteroid group than in those receiving placebo after 12 weeks [mean (SD) score on a 10-cm VAS after 12 weeks of 2.2 (2.9) vs. 4.0 (3.2), respectively]. Also, a significant decrease in joint swelling was found in the corticosteroid-treated group compared with the placebo group [1.1 (1.2) vs. 2.0 (1.3), respectively on a 10-cm VAS]. For the other reported efficacy outcomes (i.e. function [measured with both the Cochin questionnaire and the Australian/Canadian hand osteoarthritis index (AUSCAN)], grip strength, pinch strength and goniometry), no between-group differences were identified. The authors reported that no severe adverse events were observed in either treatment group.

\subsubsection{Intra-Articular Hyaluronic Acid Versus Placebo}

Three studies $(n=196)$ investigated an injection with hyaluronic acid compared with placebo in participants with CMC OA [19, 22, 24]. One trial investigated the same participants in both treatment groups by including one CMC joint in the intervention group and the contralateral joint in the placebo group [19]. All studies [risk of bias: high $(n=1)$ and unclear $(n=2)]$ showed a decrease in pain compared with baseline in both groups, but no significant between-group differences were found up to 26 weeks in two studies, while one study did not formally investigate between-group differences. Furthermore, no between-group differences were reported for the other and Meenagh et al. [26]. CI confidence interval, $d f$ degrees of freedom, $I V$ inverse variance, $m m$ millimetres, $S D$ standard deviation, $V A S$ visual analogue scale

efficacy outcomes [i.e. function (three trials), grip strength (one trial), pinch strength (two trials) and range of motion of the thumb (one trial)]. No adverse events were observed in any of the treatment groups in two trials, although one trial [24] observed adverse events in both treatment groups [5/62 in the hyaluronic acid-group (three local adverse events and two cases of surgery unrelated to study medication) and 3/61 in the placebo group (two local adverse events and one case of surgery unrelated to study medication)].

\subsubsection{Intra-Articular Corticosteroids Versus Hyaluronic Acid}

Six studies $(n=405)$ investigated a corticosteroid injection compared with hyaluronic acid in participants with CMC OA $[18,21,22,24,27,31]$. For the primary outcome-pain on VAS - all trials showed an improvement in both treatment groups. Four trials [risk of bias: high $(n=2)$ and unclear $(n=2)]$ showed no between-group difference in pain on VAS up to 26 weeks [22, 24, 27, 31], one trial (high risk of bias) described a transitory superior effect of corticosteroids at 1 and 6 months [18], and one trial (high risk of bias) concluded that the corticosteroid group initially displayed a better and faster pain relief up to 3 weeks but that hyaluronic acid was 'non-inferior' to corticosteroids thereafter [21]. Three studies [risk of bias: high $(n=1)$ and unclear $(n=2)]$ reported no betweengroup differences in self-reported function after 26 weeks $[22,24,27]$, although one trial (high risk of bias) reported temporarily more improvement in function in the corticosteroid group at 12 months of follow-up [18]. Three of four trials assessing self-reported function reported an improvement in function in both intervention groups [18, $22,27]$. No between-group differences were found for most other reported efficacy parameters [i.e. grip strength (one trial), pinch strength (one trial), range of motion of the thumb (one trial), joint pain on pressure (one trial), quality 
of life (one trial) and joint crepitation (one trial)]. However, single studies reported temporarily more improvement in grip strength in the corticosteroid group at 1 month of follow-up [18], and transitory better relief of joint swelling in the corticosteroid group [21]. In contrast, single studies also reported (transitory) beneficial effects in favour of hyaluronic acid injections in the outcomes pinch strength (temporarily more improvement after 12 weeks [22] and 24 weeks [21]), range of motion of the thumb (overall more improvement in the hyaluronic acid group [21]) and joint warmth (overall better relief of warmth in the hyaluronic acid group [21]). One trial only reported effects within each treatment group without formally comparing the two interventions for the outcomes function performance, grip strength and pinch strength [31].

Three trials reported adverse events in both treatment groups, although numbers were relatively low and equal in both groups [corticosteroid vs. hyaluronic acid groups: $4 / 28$ vs. 4/28 (Fuchs et al. [21], not related to study medication), 5/40 vs. 5/48 (Monfort et al. [27], minor side effects, including pain and local swelling following the injection), 9/65 vs. 5/62 (Mandl et al. [24], local side effects such as pain, swelling and skin and nail abnormalities, and few cases of surgery unrelated to study medication)], although no adverse events were observed in any of the treatment groups in the remaining three studies in this comparison.

\subsubsection{Other Comparisons}

One study ( $n=20$, high risk of bias) investigated intraarticular infliximab injections compared with placebo in a non-randomised pilot study in participants with erosive IP OA [20]. From each participant, the hand with the most severely affected finger joints was assigned to the intervention group; the other hand served as a control. Pain on VAS had significantly improved in the infliximab group after 12 months compared with baseline [mean (SD) 32.5 (15.1) vs. 75.3 (10.2), respectively, on a 100-mm VAS] and not in the placebo group [62.5 (20.4) vs. 50.5 (13.0)]. A formal between-group comparison was not possible due to the (known) baseline differences between the intervention and placebo groups. Pain on pressure also improved only in the infliximab group, although no differences between groups were found for the other reported efficacy outcomes (i.e. grip strength, morning stiffness and radiological score). No side effects, either local or systemic, were observed during the study.

One study ( $n=60$, unclear risk of bias) investigated intra-articular dextrose injections compared with corticosteroid injections in participants with CMC OA [23]. Pain during movement on VAS improved in both groups, with better pain relief 24 weeks after the last injection in the dextrose group compared with the corticosteroid group [mean (SD) score on a 10-cm VAS after 24 weeks of 1.2 (1.6) vs. 2.4 (1.8), respectively]. The outcomes function and joint pain on pressure also improved more in the dextrose group than in the corticosteroid group [function after 24 weeks: 1.6 (1.3) vs. 2.6 (1.5) on the Health Assessment Questionnaire Disability Index (scale 0-9, higher is worse); results for pain on pressure were only presented graphically]. No between-group differences were found for pinch strength. Three participants experienced minor adverse events (increase in joint pain, which subsided in several days), although which treatment these participants had received is unclear.

Two studies compared different frequencies or different forms of hyaluronic acid with each other in participants with CMC OA. The first study ( $n=42$, high risk of bias) compared a single injection of hyaluronic acid with either 2- or 3-weekly injections [28, 29]. Pain on VAS improved only in both groups with multiple injections and not in the group with a single injection, although no between-group differences were found after 12 weeks [mean (SD) score on a 100-mm VAS after 12 weeks of 43.1 (22.8) vs. 39.5 (28.6) vs. 29.8 (21.9), respectively, for one, two or three injections]. Minor adverse events were observed in $30 \%$ of participants in all treatment groups, involving local pain, swelling, heat and/or redness, which subsided within several hours to days. The second study ( $n=80$, unclear risk of bias) compared hyaluronic acid with a low molecular weight with that with a high molecular weight [25]. Pain on VAS improved in both treatment groups, although no between-group differences were found after 12 weeks [mean (SD) score on a 10-cm VAS after 12 weeks of 4.23 (2.90) vs. 4.03 (2.56), respectively, for the low versus high molecular weight fluid]. Minor side effects were observed in both groups, including mild pain and/or ecchymosis in the injection site (exact numbers per treatment group not specified).

\section{Discussion}

Based upon trials of variable quality, both intra-articular corticosteroid injections and intra-articular hyaluronic acid injections appear not to be more efficacious than placebo in participants with CMC OA. A pooled analysis of two trials (low and unclear risk of bias) comparing corticosteroid with placebo injections in participants with CMC OA demonstrated no between-group difference in pain up to 26 weeks. However, one trial with a low risk of bias showed that intra-articular corticosteroid injections might be more effective than placebo in relieving both pain during joint movement and joint swelling in participants with IP OA. This between-group difference also reached 
clinical significance with a minimal clinically important difference (MCID) of $0.9 \mathrm{~cm}$ on a $10-\mathrm{cm}$ VAS, based on studies in participants with OA of the lower extremities, in the absence of an MCID based on studies with patients with hand OA [12]. This finding possibly suggests different effects of intra-articular corticosteroid injections in different subsets of hand OA, although this needs confirmation since-to our knowledge-only one study to date has investigated intra-articular corticosteroids in IP OA. It is well known that CMC and IP OA are associated with a separate risk factor profile, which underlines the different nature of these two subsets of hand OA and thus the possibility of different effects of the same intervention [32]. It is possible that inflammation plays a bigger role in IP OA than it does in CMC OA, in which mechanical loading is known to be an important factor, explaining why corticosteroids might be more effective in IP OA. Several trials in this review, all with a high or unclear risk of bias, compared corticosteroid versus hyaluronic acid injections in participants with CMC OA, but no consistent beneficial effect of one intervention over the other could be demonstrated. Single studies, all judged to have a high or unclear risk of bias, investigated alternative intra-articular therapies, including infliximab versus placebo (possible beneficial effect of infliximab, although no formal comparison could be made), dextrose versus corticosteroid injections (more improvement of pain during movement, pain on pressure and function in the dextrose group), single versus two or three hyaluronic acid injections (no between-group differences) and low versus high molecular weight hyaluronic acid (no between-group differences).

Apart from local adverse events at the injection site, including local pain, swelling and skin and/or nail abnormalities, no important adverse events were reported in any of the trials, indicating that the intra-articular therapies studied in this review appear to be relatively safe. Based on the included studies, none of the intra-articular therapies appeared to be more harmful than another. However, studies were small and possibly underpowered to assess adverse events. A large meta-analysis investigating the benefits and harms of viscosupplementation for knee OA found a clinically important increase in the risk for serious adverse events, withdrawals because of adverse events, and local adverse events associated with the use of intra-articular hyaluronic acid [12]. However, dosages of hyaluronic acid used in studies in hand OA (range 5-15 mg per injection) were generally significantly lower than the dosage commonly used in the treatment of knee OA (usually 20-40 mg per injection), possibly decreasing the risk of adverse events. A network meta-analysis investigating all pharmacological interventions in knee OA reported more withdrawals due to adverse events in oral treatments (i.e. acetaminophen and NSAIDs) than in intraarticular therapies [33]. The most commonly reported adverse events among intra-articular therapies for knee OA were similar to those found in this review, i.e. transient local reactions, with similar events reported in different intra-articular therapies (corticosteroids and hyaluronic acid). Since all included studies were of a short duration, we cannot draw conclusions on long-term safety and/or potential side effects associated with repeated intra-articular injections in the finger joints.

Studies systematically evaluating the benefits and harms of intra-articular therapies in hand OA are scarce, although many studies have been performed in knee OA, with conflicting results [9, 12, 34-37]. A recently published network meta-analysis comparing the effects of all available treatments in knee OA showed that intra-articular therapies were the most effective form of treatment [33]. The authors of this meta-analysis also found that intraarticular injection with placebo had an effect on pain similar to that of an (oral) NSAID, both with an effect size of 0.3 . The intra-articular delivery mode itself thus appears to have a large positive treatment effect, which could be a true placebo response or possibly a physiological effect after injecting a fluid into a joint with a needle. The positive treatment effect of intra-articular injections in general was also confirmed by our finding that, in all placebocontrolled studies, not only the intervention group, but also the placebo group improved over time.

One of the strengths of our study was the use of a rigorous search strategy, minimizing the risk of missing eligible studies. In an attempt to reduce the risk of publication bias, we also searched for unpublished literature. This yielded two additional potentially relevant trials for inclusion, but both trials were eventually excluded, since one trial (hyaluronic acid vs. corticosteroids in CMC OA) was prematurely terminated due to adverse events in the hyaluronic acid group (trial registration number ISRCTN63038599), and results from the other (hyaluronic acid vs. placebo in CMC OA) were unobtainable because the pharmaceutical company funding the trial did not pursue the indication (trial registration number NCT00423371). Furthermore, through database searching we retrieved a trial comparing a thumb splint alone versus a single intra-articular corticosteroid injection in the CMC joint in addition to a thumb splint, which did not fulfil our inclusion criteria since it was only presented as an abstract at a conference meeting more than 5 years ago [38]. In this trial, no sustained benefit from a corticosteroid injection in addition to wearing a thumb splint could be demonstrated. Despite rigorous searching, no full trial report of this study could be identified. The exclusion of the three abovementioned trials has possibly introduced a risk of publication bias. However, we believe it is unlikely that 
including these three studies would have led to major implications for the conclusions of our review, since we found no clear positive effect of any of the intra-articular therapies studied.

An important limitation is the poor methodological quality of many of the included trials, which is (partly) reflected by the fact that many of the included studies were judged as having an overall high risk of bias. Moreover, many studies did not provide data eligible for meta-analysis (e.g. authors presented data only in figures or presented point estimates without a measure of variance). We decided to only perform meta-analysis if the study data were clinically and statistically sufficiently homogeneous and/or the risk of bias was judged to be low enough to produce reliable results. This decision was made based on previous reports that different forms of bias can have a major impact on the estimates of treatment benefits in osteoarthritis trials [39-41]. Consequently, pooled analysis was only possible in the comparison of corticosteroid injections versus placebo in participants with CMC OA. Although this comparison consisted of three trials, only two of these were included in the meta-analysis, since the third trial only presented data in figures and its trial population was also too different from that of the other two studies (i.e. participants were only included when they had previously experienced at least a mild to moderate improvement after a corticosteroid injection in the CMC joint). However, we believe that further studies with higher methodological quality are not likely to substantially change the conclusions of this review. Confounding from biases across studies would have likely favoured the intraarticular treatment, and correcting for these biases is thus unlikely to change the direction of results towards a benefit of intra-articular treatment with corticosteroids or hyaluronic acid for hand OA compared with placebo. Our decision not to include all available studies in a metaanalysis likely explains why our findings differ slightly from those of a recently published systematic review evaluating the effectiveness of intra-articular corticosteroids and hyaluronic acid in participants with thumb-base OA [13].

It is known that generalizing results from the specialized and highly controlled setting of an RCT to real-life settings can sometimes be troublesome, and it could be argued that (the lack of) treatment effects found in the trials in our review might not be consistent with experiences from clinical practice. However, we believe that evidence from RCTs, provided that such a study is well-executed, is the most reliable form of evidence we have, since it accounts for several important forms of bias. Reasons that clinicians might see a treatment effect from any form of intra-articular therapy despite it not being found in the studies in this review, are, for example, a 'regression to the mean' effect because patients come to their treating physician when complaints are at the maximum level, and the placebo effect.

We aimed to include all possible intra-articular therapies for hand $\mathrm{OA}$ in this review, and our extensive database search also retrieved a few studies investigating prolotherapy in hand OA. Prolotherapy is a form of treatment in which repeated injections with an irritating substance are given around the joint (peri-articular). A commonly used substance for this purpose is dextrose, which is the compound that is also studied by one of the included studies [23]. However, since most studies investigating prolotherapy did not clearly indicate whether the solution was only injected peri-articularly or whether it entered the intra-articular space, we excluded studies investigating prolotherapy unless they clearly specified that the investigated solution was injected intra-articularly. This led to the exclusion of one RCT comparing dextrose prolotherapy $(n=13)$ versus placebo $(n=14)$ in participants with finger and thumb OA, reporting that pain at rest and with grip improved more in the dextrose group, although not significantly, and pain with movement as well as finger flexion improved significantly more in the dextrose group [42].

\section{Conclusions}

For patients with CMC OA, intra-articular injections with corticosteroids or hyaluronic acid do not seem to be more effective than placebo, although in the short term these treatments are probably not associated with important adverse events other than local side effects. Despite the apparent beneficial safety profile of intra-articular treatments, at least in short-term use, which would suggest an interesting treatment option for clinicians treating elderly patients, the lack of efficacy discourages the use of these treatments for hand OA. More well-performed studies investigating these therapies in this patient group are unlikely to substantially change this conclusion. Findings from one well-performed study suggested that intra-articular corticosteroid injections might be effective compared with placebo for patients with IP OA, although this finding needs to be confirmed in future studies.

Acknowledgments The authors thank N. Spolidoro, K. Lewis and L. Mandl for providing additional information about their studies on request.

\section{Compliance with Ethical Standards}

Conflicts of interest FK, RR and JS have no conflicts of interest. MK received financial support from Pfizer, Abbvie, Servier, TI Pharma, all of which were paid to the institution.

Funding None. 
Open Access This article is distributed under the terms of the Creative Commons Attribution-NonCommercial 4.0 International License (http://creativecommons.org/licenses/by-nc/4.0/), which permits any noncommercial use, distribution, and reproduction in any medium, provided you give appropriate credit to the original author(s) and the source, provide a link to the Creative Commons license, and indicate if changes were made.

\section{References}

1. Dahaghin S, Bierma-Zeinstra SM, Ginai AZ, Pols HA, Hazes JM, Koes BW. Prevalence and pattern of radiographic hand osteoarthritis and association with pain and disability (the Rotterdam study). Ann Rheum Dis. 2005;64:682-7.

2. Michon M, Maheu E, Berenbaum F. Assessing health-related quality of life in hand osteoarthritis: a literature review. Ann Rheum Dis. 2011;70:921-8.

3. Zhang Y, Niu J, Kelly-Hayes M, Chaisson CE, Aliabadi P, Felson DT. Prevalence of symptomatic hand osteoarthritis and its impact on functional status among the elderly: The Framingham Study. Am J Epidemiol. 2002;156:1021-7.

4. Kloppenburg M. Hand osteoarthritis-nonpharmacological and pharmacological treatments. Nat Rev Rheumatol. 2014;10:242-51.

5. Zhang W, Doherty M, Leeb BF, Alekseeva L, Arden NK, Bijlsma $\mathrm{JW}$, et al. EULAR evidence based recommendations for the management of hand osteoarthritis: report of a Task Force of the EULAR Standing Committee for International Clinical Studies Including Therapeutics (ESCISIT). Ann Rheum Dis. 2007;66:377-88.

6. Wolf JM, Delaronde S. Current trends in nonoperative and operative treatment of trapeziometacarpal osteoarthritis: a survey of US hand surgeons. J Hand Surg Am. 2012;37:77-82.

7. Grillet B, Dequeker J. Intra-articular steroid injection. A riskbenefit assessment. Drug Safety. 1990;5:205-11.

8. Hochberg MC, Altman RD, April KT, Benkhalti M, Guyatt G, McGowan J, et al. American College of Rheumatology 2012 recommendations for the use of nonpharmacologic and pharmacologic therapies in osteoarthritis of the hand, hip, and knee. Arthritis Care Res. 2012;64:465-74.

9. Bellamy N, Campbell J, Robinson V, Gee T, Bourne R, Wells G. Viscosupplementation for the treatment of osteoarthritis of the knee. Cochrane Database Syst Rev. 2006;2:CD005321.

10. Fam H, Bryant JT, Kontopoulou M. Rheological properties of synovial fluids. Biorheology. 2007;44:59-74.

11. Hunter DJ. Viscosupplementation for osteoarthritis of the knee. N Engl J Med. 2015;372:1040-7.

12. Rutjes AW, Juni P, da Costa BR, Trelle S, Nuesch E, Reichenbach S. Viscosupplementation for osteoarthritis of the knee: a systematic review and meta-analysis. Ann Intern Med. 2012;157:180-91.

13. Trellu S, Dadoun S, Berenbaum F, Fautrel B, Gossec L. Intraarticular injections in thumb osteoarthritis: a systematic review and meta-analysis of randomized controlled trials. Joint Bone Spine. 2015 [Epub ahead of print].

14. Altman RAG, Appelrouth D, Bloch D, Borenstein D, Brandt K, Brown C, Cooke TD, Daniel W, Gray R, et al. The American College of Rheumatology criteria for the classification and reporting of osteoarthritis of the hand. Arthritis Rheum. 1990;33:1601-10.

15. Higgins JPT, Sterne JAC. Assessing risk of bias in included studies. In: Cochrane handbook for systematic reviews of interventions version 5.1.0 [updated March 2011]. The Cochrane Collaboration.
2011. http://handbook.cochrane.org/chapter_8/8_assessing_risk_ of_bias_in_included_studies.htm. Accessed June 2015.

16. Kloppenburg M, Bøyesen P, Visser W, Haugen IK, Boers M, Boonen A, et al. Report from the OMERACT hand osteoarthritis workshop: set of core domains and preliminary set of instruments for use in clinical trials and observational studies. J Rheumatol. 2015 [Epub ahead of print].

17. Wan X, Wang W, Liu J, Tong T. Estimating the sample mean and standard deviation from the sample size, median, range and/or interquartile range. BMC Med Res Methodol. 2014;14:135-47.

18. Bahadir C, Onal B, Dayan VY, Gurer N. Comparison of therapeutic effects of sodium hyaluronate and corticosteroid injections on trapeziometacarpal joint osteoarthritis. Clin Rheumatol. 2009;28:529-33.

19. Figen AF, Ustun N. The evaluation of efficacy and tolerability of Hylan G-F 20 in bilateral thumb base osteoarthritis: 6 months follow-up. Clin Rheumatol. 2009;28:535-41.

20. Fioravanti A, Fabbroni M, Cerase A, Galeazzi M. Treatment of erosive osteoarthritis of the hands by intra-articular infliximab injections: a pilot study. Rheumatol Int. 2009;29:961-5.

21. Fuchs S, Monikes R, Wohlmeiner A, Heyse T. Intra-articular hyaluronic acid compared with corticoid injections for the treatment of rhizarthrosis. Osteoarthr Cartilage. 2006;14:82-8.

22. Heyworth BE, Lee JH, Kim PD, Lipton CB, Strauch RJ, Rosenwasser MP. Hylan versus corticosteroid versus placebo for treatment of basal joint arthritis: a prospective, randomized, double-blinded clinical trial. J Hand Surg Am. 2008;33:40-8.

23. Jahangiri A, Moghaddam FR, Najafi S. Hypertonic dextrose versus corticosteroid local injection for the treatment of osteoarthritis in the first carpometacarpal joint: a double-blind randomized clinical trial. J Orthop Sci. 2014;19:737-43.

24. Mandl LA, Wolfe S, Daluiski A, Hotchkiss RN, Lyman SL, Katz JN. A randomized controlled trial of hylan G-F 20 for the treatment of carpometacarpal osteoarthritis. Arthritis Rheum. 2012;64:S475-6.

25. Massarotti M, Crotti C, Ughi N, Uboldi F, Belloli L, Marasini B. Comparison of two different intra-articular hyaluronic acid compounds for carpo-metacarpal joint osteoartrhitis. Ann Rheum Dis. 2013;71:695.

26. Meenagh GK, Patton J, Kynes C, Wright GD. A randomised controlled trial of intra-articular corticosteroid injection of the carpometacarpal joint of the thumb in osteoarthritis. Ann Rheum Dis. 2004;63:1260-3.

27. Monfort J, Rotes-Sala D, Segales N, Montanes FJ, Orellana C, Llorente-Onaindia J, et al. Comparative efficacy of intra-articular hyaluronic acid and corticoid injections in osteoarthritis of the first carpometacarpal joint: results of a 6-month single-masked randomized study. Joint Bone Spine. 2015;82:116-21.

28. Roux C, Fontas É, Breuil V, Brocq O, Albert C, Euller-Ziegler L. Injection of intra-articular sodium hyaluronidate (Sinovial) into the carpometacarpal joint of the thumb (CMC1) in osteoarthritis. A prospective evaluation of efficacy. Joint Bone Spine. 2007;74:368-72.

29. Roux C, Fontas É, Breuil V, Brocq O, Albert C, Euller-Ziegler L. Injection of intra-articular sodium hyaluronate into the carpometacarpal joint of the thumb (CMC1) in osteoarthritis: a prospective evaluation of efficacy [Infiltration intra-articulaire d'acide hyaluronique dans l'articulation trapézométacarpienne du pouce: évaluation prospective de l'efficacité dans la rhizarthrose]. Rev Rhum. 2007;74:660-5.

30. Spolidoro NDO, Machado FS, Oliveira HAV, Natour J, Furtado RNV. Interphalangeal intra-articular injection with triamcinolone hexacetonide: Assessment of effectiveness in hand osteoarthritis. Arthritis Rheum. 2013;65(Suppl 10):2141.

31. Stahl S, Karsh-Zafrir I, Ratzon N, Rosenberg N. Comparison of intraarticular injection of depot corticosteroid and hyaluronic acid 
for treatment of degenerative trapeziometacarpal joints. J Clin Rheumatol. 2005;11:299-302.

32. Kloppenburg M, Kwok WY. Hand osteoarthritis-a heterogeneous disorder. Nat Rev Rheumatol. 2012;8(1):22-31.

33. Bannuru R, Schmid C, Kent D, Vaysbrot E, Wong J, McAlindon T. Comparative effectiveness of pharmacologic interventions for knee osteoarthritis. Ann Intern Med. 2015;162:46-55.

34. Bellamy N, Campbell J, Robinson V, Gee T, Bourne R, Wells G. Intraarticular corticosteroid for treatment of osteoarthritis of the knee. Cochrane Database Syst Rev. 2006;2:CD005328.

35. Lo GH, LaValley M, McAlindon T, Felson DT. Intra-articular hyaluronic acid in treatment of knee osteoarthritis: a meta-analysis. JAMA. 2003;290:3115-21.

36. Wang CT, Lin J, Chang CJ, Lin YT, Hou SM. Therapeutic effects of hyaluronic acid on osteoarthritis of the knee. A meta-analysis of randomized controlled trials. J Bone Joint Surg Am. 2004;86A:538-45.

37. Wang $\mathrm{F}, \mathrm{He} \mathrm{X}$. Intra-articular hyaluronic acid and corticosteroids in the treatment of knee osteoarthritis: a meta-analysis. Exp Ther Med. 2015;9:493-500.

38. Negi A, Wilding J, Kallarackal G, O'Sullivan MM, Harvey JN, Amos N. Primary osteoarthritis of base of the thumb: a randomised controlled trial of intra-articular steroid vs thumb splint. Rheumatology. 2006;45:I74.

39. Nüsch E, Reichenbach S, Trelle S, Rutjes AW, Liewald K, Sterchi R, et al. The importance of allocation concealment and patient blinding in osteoarthritis trials: a meta-epidemiologic study. Arthritis Rheum. 2009;61(12):1633-41.

40. Nüsch E, Trelle S, Reichenbach S, Rutjes AW, Burgi E, Scherer $M$, et al. The effects of excluding patients from the analysis in randomised controlled trials: meta-epidemiological study. BMJ. 2009;339:b3244.

41. Nüsch E, Trelle S, Reichenbach S, Rutjes AW, Tschannen B, Altman DG, et al. Small study effects in meta-analyses of osteoarthritis trials: meta-epidemiological study. BMJ. 2010;341:c3515.

42. Reeves KD, Hassanein K. Randomized, prospective, placebocontrolled double-blind study of dextrose prolotherapy for osteoarthritic thumb and finger (DIP, PIP, and trapeziometacarpal) joints: evidence of clinical efficacy. J Altern Complem Med. 2000;6(4):311-20. 